\title{
Efecto de una dieta personalizada en mujeres embarazadas con sobrepeso u obesidad
}

\author{
Personalized diet effect in pregnant \\ obese or overweight women
}

\begin{abstract}
Objectives: To determine if the implementation of a personalized diet for three months improves the nutritional status of pregnant women with overweight and obesity of the UMF No. 56. Methodology: Prospective longitudinal study in 53 overweight or obese pregnant women to whom which were given a specific diet, according to its particular characteristics; were evaluated the characteristics of the right diet, 24 hours recall, frequency of food consumption, weight and biochemical indicators. The real, ideal and projected weight of the patients was compared. McNemar test and Kuskal-Wallis was applied with confidence interval of $95 \%$. Results: the BMI pre-gestational average was $28.5 \mathrm{~kg} / \mathrm{m} 2$. At the end of the study pregnant women improved the characteristics of the right diet: the complete one $(9.4 \% \mathrm{vs}$ $60.4 \%$ ), the percentage of adequacy of lipids (7.5\% vs $45.3 \%$ ) and increased in active physical activity (22.6\% vs $54.7 \%$ ). Ideal gestational weight wasn't reached, but there was a weight control reflected in a projected weight higher than real in each intervention. No significant changes in the biochemical indicator ( $p>0.05)$. Conclusions: The implementation of a personalized diet improved the nutritional status of overweight or obese pregnant women in their eating patterns and gestational weight control. Keywords: gestational weight gain; overweight; obesity; pregnancy; weight.
\end{abstract}

\section{INTRODUCCIÓN}

El sobrepeso y la obesidad son una acumulación excesiva de tejido adiposo perjudicial para la salud (1). Según la Encuesta Nacional de Salud y Nutrición 2012 (ENSANUT 2012), en México 35.3\% de las mujeres de 20 a 49 años tiene sobrepeso y $35.2 \%$ obesidad (2). El periodo de embarazo representa el periodo más intenso de crecimiento y desarrollo; cómo se presenta depende de factores que en su mayoría son posibles modificar, entre estos destaca el estado nutricional (3). Las recomendaciones de ganancia de peso gestacional (GPG) están relacionadas con el nacimiento de niños con peso saludable (3500 a $4000 \mathrm{~g}$ ). El peso pregestacional (PP) influye en el aumento de peso durante el embarazo, cuanto mayor sea el PP menor será el aumento necesario durante el embarazo debido a que puede usarse una porción de los depósitos de energía para apoyar el crecimiento fetal; las guías actuales del Instituto de Medicina 2009 (IOM) de Estados Unidos, recomiendan una GPG de 7-11.5 kg en mujeres con sobrepeso y $9.5 \mathrm{~kg}$ para las
Karen Vianey Sandoval G. (1) Efrén René Nieves R. (2) Miguel Ángel Luna R. (3)

(1) Servicio Social Profesional de Nutrición y Ciencia de los Alimentos. Unidad de Medicina Familiar Número 56 León, Guanajuato, Mexico.

(2) Educación e Investigación en Salud. León, Guanajuato, Mexico.

3) Investigación Clínica. Universidad Iberoamericana León. León, Guanajuato, Mexico.

Dirigir la correspondencia a: Profesor Efrén René Nieves $R$. Educación e Investigación en Salud UMF 56, León, Guanajuato

Correo: efren.nieves@imss.gob.mx Teléfono trabajo: 4777781110 ext. 31470 y 31479

Este trabajo fue recibido el 25 de Enero de 2016 aceptado con modificaciones el 3 de Abril de 2016 y aceptado para ser publicado el 24 de Agosto e 2016.

mujeres con obesidad (4).

Se ha demostrado que mujeres con GPG mayor a la recomendada presentan incremento en el riesgo de hipertensión asociada con el embarazo, diabetes mellitus gestacional (DMG), complicaciones durante el trabajo de parto, macrosomía fetal, falla en la lactancia, retención de peso posparto y subsecuente desarrollo de obesidad (4-6).

El estado nutricional de las mujeres embarazadas con sobrepeso u obesidad está vinculado íntimamente con la alimentación que siga la persona (7). El consumo elevado de alimentos de alto contenido calórico, predispone al aumento de peso excesivo; en el embarazo el alto consumo de alimentos con alto contenido glucémico a la macrosomía fetal; una dieta con alimentos de bajo contenido glucémico aunque no tiene efecto en el peso al nacer, en un grupo en riesgo de macrosomía fetal, sí tiene un efecto positivo sobre la GPG (8). La evaluación realizada por Renault y col. muestra que una GPG elevada está mayormente relacionada con el consumo 
de alimentos con azúcares agregados, que con la ingesta de grasas saturadas y exceso de proteínas (9).

Existen pocos estudios que muestren el impacto de la implementación de un plan de alimentación sobre el control de la GPG, no obstante, técnicas utilizadas en intervenciones exitosas involucran actividad física y asesoramiento de un nutriólogo, complementando con motivación en el control del peso, retroalimentación sobre el progreso y seguimiento continuo (10).

El propósito de este estudio fue, determinar si el estado nutricional de mujeres embarazadas con sobrepeso u obesidad de la UMF No. 56 mejora con la implementación de una dieta personalizada.

\section{PLANTEAMIENTO DEL PROBLEMA}

¿La implementación de una dieta personalizada durante tres meses mejora el estado nutricional en mujeres embarazadas con sobrepeso y obesidad de la unidad de medicina familiar (UMF) No. 56 de León, Guanajuato, México?

\section{OBJETIVOS}

General: Determinar si la implementación de una dieta personalizada durante tres meses mejora el estado nutricional de mujeres embarazadas con sobrepeso y obesidad de la UMF No. 56.

Específicos: Evaluar las características de la dieta de las mujeres embarazadas con sobrepeso y obesidad mediante una encuesta de recordatorio de $24 \mathrm{hrs}$ al inicio y mensualmente durante tres meses, y la frecuencia de consumo de alimentos al inicio y al final del estudio.

Evaluar los indicadores antropométricos de peso, talla, peso previo a la gestación, peso esperado de acuerdo al IMC previo a la gestación y edad de gestación (peso ideal), y porcentaje de peso esperado de acuerdo al IMC previo a la gestación y edad de gestación, mensualmente durante 3 meses.

Evaluar los indicadores bioquímicos de glucosa, hemoglobina, y hematocrito antes y después de los tres meses de tratamiento.

\section{HIPÓTESIS}

La implementación de una dieta personalizada mejora el estado nutricio de las mujeres embarazadas con sobrepeso y obesidad al final del estudio.

\section{SUJETOS Y MÉTODOS}

Se realizó un estudio prospectivo longitudinal a 53 embarazadas diagnosticadas con sobrepeso y obesidad, que acudieron a la Unidad de Medicina Familiar No. 56 de León, Guanajuato, a las que se les proporcionó una dieta personalizada durante el período de mayo a noviembre de 2015 .

Se determinó una muestra de 66 pacientes mediante el programa Power Analysis and Sample Size 2008, para demostrar que con la dieta implementada mejoraría el estado nutricio del $30 \%$ de las mujeres embarazadas con sobrepeso u obesidad. Se consideraron estudios previos donde referían que un programa de restricción de ganancia de peso en mujeres embarazadas obesas benefició a un 31.4\% de las mujeres (11). Con una potencia de prueba de $80 \%$ y un nivel de confianza de $95 \%$, la muestra calculada requirió de 51 pacientes, sin embargo, se incrementó un 30\% para sustituir a quiénes abandonaran el estudio.

En el estudio participaron 66 embarazadas seleccionadas por simple disponibilidad que fueron enviadas por los médicos familiares de los consultorios 1 al 10 del horario matutino al servicio de nutrición; a las pacientes embarazadas con un mínimo de 16 y máximo de 32 semanas de gestación diagnosticadas con sobrepeso u obesidad mediante IMC pregestacional mayores de 18 años, se les invitó a participar en el estudio, se les explicaron los objetivos y procedimientos de este, y a quienes aceptaron participar se les pidió firmar una carta de consentimiento informado (ANEXO 1). Posteriormente se obtuvo por interrogatorio la historia clínico-nutricia del paciente, (ANEXO 2).

El protocolo de investigación se llevó a cabo de acuerdo a los lineamientos de Declaración de Helsinki y sus modificaciones posteriores, de la NOM-012-SSA3-2012 y del Reglamento de la Ley General de Salud. El presente estudio fue aprobado por el Comité local de Investigación y Bioética del Instituto Mexicano del Seguro Social.

No se incluyeron en el estudio a embarazadas con productos múltiples, embarazo de alto riesgo, o diagnóstico de DMG. Se excluyeron a pacientes con parto pretérmino, que no asistieron a una o más consultas de nutrición, o que presentaron uno o más de los criterios de no inclusión a lo largo del estudio.

Posteriormente se evaluaron las características de la dieta mediante la aplicación de una encuesta de recordatorio de 24 horas de pasos múltiples y frecuencia de consumo de alimentos (ANEXO 3), al inicio y mensualmente durante tres meses para el R24h y al inicio y el final para la frecuencia de consumo de alimentos, los cuales se compararon con las características de la dieta correcta según lo estipulado en la NOM 043 SSA2 2012: (12)

Completa: que contuviera todos los nutrientes. Se recomienda incluir en cada comida alimentos de los 3 grupos

Equilibrada: que los nutrimentos guarden las proporciones apropiadas entre sí.

Adecuada: acorde con los gustos y la cultura de quien la consume y ajustada a sus recursos económicos, sin que ello signifique que se deban sacrificar sus otras características.

Higiénica: que su consumo habitual no implique riesgos para la salud porque está exenta de microorganismos patógenos, toxinas, contaminantes; que se consuma con mesura y que no aporte cantidades excesivas de ningún componente o nutrimento.

Suficiente: que cubra las necesidades de todos los nutrimentos, de tal manera que el sujeto adulto tenga una buena nutrición y un peso saludable.

Variada: que, de una comida a otra, incluya alimentos diferentes de cada grupo.

Para la identificación con mayor precisión de los alimentos consumidos, se utilizaron imágenes de alimentos del Kit para consultas de 103 alimentos marca Nutrikit $\circledR_{\text {, basado en el }}$ Sistema Mexicano de Alimentos Equivalentes $4^{\mathrm{a}}$ ed, así como también utensilios de cocina como tazas, cucharas, vasos y platos de diversos tamaños.

Se realizaron las mediciones antropométricas al inicio y mensualmente hasta finalizar el estudio, se utilizaron las técnicas antropométricas de Lohman (13), para el peso se utilizó una báscula mecánica marca BAME® modelo 425, con precisión de $100 \mathrm{~g}$ y capacidad de $160 \mathrm{~kg}$.

Para la talla, se usó la misma báscula mecánica con estadímetro de capacidad de $200 \mathrm{~cm}$ y precisión de $1 \mathrm{~mm}$, se registró la medición en metros. Ambas mediciones se registraron en la hoja de antropometría, (ANEXO 4).

Para obtener el peso ideal, de acuerdo a las semanas de gestación, se le preguntó a la paciente su peso antes del embarazo, o bien, se consideró el peso registrado en su Cartilla 
Nacional de Salud o en registros médicos previos, a este peso se le sumó el aumento de peso durante el embarazo según el IMC pregestacional, tomando como referencia las tablas de Casanueva y colaboradores 2008 (ANEXO 5).

Con el peso ideal se calculó el porcentaje de peso esperado, de acuerdo con el IMC previo a la gestación y la edad de gestación (\%PelMCpgEg) mediante la siguiente fórmula: (14)

\section{\%PelMCpgEg = (peso real kg / PelMCpgEg kg) $\times 100$}

Con los datos anteriores, se realizó una proyección del peso de las embarazadas si no se hubiera implementado una dieta personalizada en relación al peso que tenía inicialmente, multiplicando el peso real por el \%PelMCpgEg entre 100, obteniendo de esta manera el peso proyectado.

Los estudios bioquímicos de glucosa, hematocrito y hemoglobina se realizaron en el laboratorio de la UMF No. 56, al inicio y a los tres meses de implementación de la dieta, para lo cual fue necesario que la persona se encontrara en ayuno de 8 horas. Dichos resultados fueron registrados en la hoja de análisis bioquímicos, (ANEXO 4).

Se evaluaron que los resultados de dichas pruebas se encontraran entre los siguientes valores:

\begin{tabular}{ll}
\hline Determinación & Rangos normales \\
\hline Hemoglobina & Segundo trimestre e inicio del tercero: \\
& $10-13 \mathrm{mg} / \mathrm{dL}$ \\
& Término del tercer trimestre: $13-15 \mathrm{mg} / \mathrm{dL}$ \\
Hematocrito & Segundo trimestre e inicio del tercero: \\
& $32.5-41.0 \%$ \\
& Término del tercer trimestre: $37-48 \%$ \\
\hline
\end{tabular}

Química sanguínea Zeman-Nay, 1996 (14)

Al finalizar estas evaluaciones, la PLNCA determinó el cálculo del gasto energético estimado (GEE) de cada paciente con la fórmula: (15)

GEE $=448-(7.95 \times$ edad en años $)+$ Actividad física $X(11.4$ $x$ peso en $\mathrm{kg}+619 \mathrm{x}$ estatura en $\mathrm{m}$ )

El peso utilizado fue el peso previo a la gestación: La actividad física se consideró de la siguiente manera (ANEXO 6): (15)

\begin{tabular}{ll}
\hline Actividad & Nivel de actividad física \\
\hline Sedentaria & 1.0 a 1.39 \\
Poco activa & 1.4 a 1.59 \\
Activa & 1.6 a 1.89 \\
Muy activa & 1.9 a 2.5 \\
\hline
\end{tabular}
(16)

El gasto energético total (GET) se calculó con la fórmula:

$\mathrm{GET}=\mathrm{GEE}$ + Factor embarazo + Depósito de energía

El factor embarazo utilizado fue de $160 \mathrm{kcal}$ en el segundo trimestre y $272 \mathrm{kcal}$ en el tercero tanto en sobrepeso y obesidad. Para el segundo y tercer trimestre el depósito de energía es de $180 \mathrm{kcal}$ (16).

La distribución de macronutrimentos empleada fue de 10-12\% de proteína (+10.7 g/día), lípidos 30-35\% e hidratos de carbono 50-55\% (principalmente alimentos con bajo índice glucémico) (17). A cada paciente se le proporcionó un formato con las raciones de alimentos que debía consumir a lo largo del mes, (ANEXO 7).

A las gestantes se les citó mensualmente para verificar el cumplimiento del plan de alimentación mediante la aplicación de la encuesta de recordatorio de 24 horas y además se pesaron para comparar el peso ideal de las pacientes, con el peso proyectado y el peso real para de esta manera conocer si los aumentos de peso gestacional van siendo adecuados. Los datos obtenidos serán registrados en sus respectivas hojas de registro.

Para el análisis estadístico de las variables cuantitativas se utilizaron medias, desviación estándar e intervalo de confianza, se utilizó prueba de Kruskal Wallis para la identificar si había diferencia entre las medianas: del peso ideal, real y proyectado. Para el análisis estadístico se utilizaron las tablas de porcentajes o gráficas de distribución de frecuencia utilizando prueba de McNemar. El programa estadístico utilizado fue NCSS 2007 con un nivel de confianza de 95\%.

\section{RESULTADOS}

Se intervinieron 53 mujeres embarazadas con sobrepeso u obesidad con un promedio de edad de 26.7 años \pm 5.3 , peso $70.8 \mathrm{~kg} \pm 9.4$, talla $1.57 \mathrm{~m} \pm 0.05$, e IMC pregestacional de $28.5 \pm 2.9$. Las características generales de las embarazadas: laborales, estado civil, escolaridad, antecedentes heredofamiliares patológicos, se encuentran en cuadro 1.

Destacan que la mayor parte de ellas son casadas, la frecuencia mayor de escolaridad es de secundaria (poco más del $50 \%$ ), el $41.5 \%$ tiene antecedentes de DM2; poco más de $3 / 4$ partes tiene sobrepeso y las demás obesidad de acuerdo al IMC pregestacional.

Todas las características de la dieta mejoraron ( $p<0.0067)$, excepto la de suficiente. La característica de la dieta correcta que tuvo mejor cambio fue la completa con $51 \%$ de diferencia, (tabla 1).

En todos los macronutrimentos y la energía, se incrementó la frecuencia de gestantes con porcentaje de adecuación recomendada de $90-110 \%$, debido a que algunos de los que tenían insuficiente o excesivo, pasaron al porcentaje recomendado. El único que tuvo diferencia significativa fue lípidos.

El alimento que mayormente mejoró su frecuencia de consumo fueron las verduras con un aumento de $26.5 \%$ y las frutas con $15 \%$. Por otra parte, el grupo de los azúcares es el que representó mayor disminución con 15.1\%, (tabla 3).

En actividad física (tabla 4), 24.5\% de las pacientes dejaron de tener actividad sedentaria, y el porcentaje de mujeres con actividad física activa del inicio al final incrementó $32.1 \%$. No se presentaron casos de muy activa ni al inicio ni al final.

El promedio de peso entre cada intervención fue $0.7 \mathrm{~kg}$ para el peso ideal, $1.8 \mathrm{~kg}$ para el real y $2.7 \mathrm{~kg}$ para el proyectado. Entre la primera y segunda intervención fue cuando hubo un aumento mayor de peso real. No hubo significancia estadística entre el peso ideal, real y proyectado de acuerdo a las semanas de gestación, (tabla 5).

Las mediciones del peso que se realizaron, se observó que las medianas del peso real estuvieron más cercanos al ideal y se alejaron más del proyectado, (tabla 5).

Las gráficas 1 y 2 muestran el promedio de peso de las pacientes de acuerdo a las semanas de gestación; se observa en la gráfica 1 que los promedios del peso real y proyectado se acercan en las semanas 20 y 24, sin embargo, logran separarse en la semana 28 , es menor el peso real y se acerca al ideal. En la gráfica 2 se observa que los promedios del peso real e ideal, no se acercaron al peso proyectado, y ambos se acercan 


\section{CUADRO 1}

Características generales de las pacientes intervenidas.

\begin{tabular}{|c|c|}
\hline Trabajaban & $\begin{array}{l}\text { Sí } 49 \% \\
\text { No } 51 \%\end{array}$ \\
\hline \multicolumn{2}{|l|}{ Estado civil } \\
\hline Casadas & $83 \%$ \\
\hline Unión libre & $17 \%$ \\
\hline \multicolumn{2}{|l|}{ Escolaridad } \\
\hline Primaria & $13.2 \%$ \\
\hline Secundaria & $56.6 \%$ \\
\hline Bachillerato & $26.4 \%$ \\
\hline Licenciatura & $3.8 \%$ \\
\hline \multicolumn{2}{|l|}{$\mathrm{AHF}^{*}$} \\
\hline $\mathrm{DM} 2$ & $41.5 \%$ \\
\hline HTA & $9.4 \%$ \\
\hline $\mathrm{DM} 2+\mathrm{HTA}$ & $15 \%$ \\
\hline Obesidad & $33.9 \%$ \\
\hline \multicolumn{2}{|c|}{ Clasificación IMC pregestacional } \\
\hline Sobrepeso & $77.4 \%$ \\
\hline Obesidad I & $18.9 \%$ \\
\hline Obesidad II & $1.8 \%$ \\
\hline Obesidad III & $1.8 \%$ \\
\hline
\end{tabular}

TABLA 1

Porcentaje de pacientes con características de la dieta correcta al inicio y al final del estudio.

\begin{tabular}{|c|c|c|c|c|c|}
\hline \multirow[t]{2}{*}{ Características de la dieta } & \multicolumn{2}{|c|}{ Inicio } & \multicolumn{2}{|c|}{ Final } & \multirow[t]{2}{*}{$\mathrm{p}(\mathrm{x} 2 \mathrm{McNemar})$} \\
\hline & $n$ & $\%$ & $\mathrm{n}$ & $\%$ & \\
\hline Completa & 5 & 9.4 & 32 & 60.4 & $<0.0001$ \\
\hline Variada & 23 & 43.4 & 47 & 88.7 & $<0.0001$ \\
\hline Higiénica & 37 & 69.8 & 51 & 96.2 & 0.0005 \\
\hline Suficiente & 22 & 41.5 & 24 & 45.3 & 0.7055 \\
\hline Equilibrada & 18 & 33.9 & 31 & 58.5 & 0.0067 \\
\hline Adecuada & 12 & 22.6 & 41 & 77.3 & $<0.0001$ \\
\hline
\end{tabular}

TABLA 2

Porcentaje de adecuación de los macronutrimentos y energía de las pacientes al inicio y al final del estudio.

\begin{tabular}{|c|c|c|c|c|c|c|c|}
\hline $\begin{array}{l}\text { Macronutrimentos } \\
\text { y energía }\end{array}$ & $\begin{array}{l}<90 \\
\mathrm{n}(\%)\end{array}$ & $\begin{array}{c}\text { Inicio } \\
90-110 \\
\text { n (\%) }\end{array}$ & $\begin{array}{l}>110 \\
\mathrm{n}(\%)\end{array}$ & $\begin{array}{l}<90 \\
\mathrm{n}(\%)\end{array}$ & $\begin{array}{c}\text { Final } \\
90-110 \\
\text { n (\%) }\end{array}$ & $\begin{array}{l}>110 \\
\mathrm{n}(\%)\end{array}$ & $p\left(x^{2}\right)$ \\
\hline $\mathrm{HC}$ & $\begin{array}{c}29 \\
54.7 \%)\end{array}$ & $\begin{array}{c}12 \\
(22.6 \%)\end{array}$ & $\begin{array}{c}12 \\
(22.6 \%)\end{array}$ & $\begin{array}{c}16 \\
(30.2 \%)\end{array}$ & $\begin{array}{c}35 \\
(66 \%)\end{array}$ & $\begin{array}{c}2 \\
(3.8 \%)\end{array}$ & $\begin{array}{c}0.785419 \\
(1.729136)\end{array}$ \\
\hline Proteínas & $\begin{array}{c}30 \\
(56.6 \%)\end{array}$ & $\begin{array}{c}14 \\
(26.4 \%)\end{array}$ & $\begin{array}{c}9 \\
(17 \%)\end{array}$ & $\begin{array}{c}17 \\
(32.1 \%)\end{array}$ & $\begin{array}{c}32 \\
(60.4 \%)\end{array}$ & $\begin{array}{c}4 \\
(7.5 \%)\end{array}$ & $\begin{array}{c}0.197016 \\
(6.028688)\end{array}$ \\
\hline Lípidos & $\begin{array}{c}21 \\
(39.6 \%)\end{array}$ & $\begin{array}{c}4 \\
(7.5 \%)\end{array}$ & $\begin{array}{c}28 \\
(52.8 \%)\end{array}$ & $\begin{array}{c}23 \\
(43.4 \%)\end{array}$ & $\begin{array}{c}24 \\
(45.3 \%)\end{array}$ & $\begin{array}{c}6 \\
(11.3 \%)\end{array}$ & $\begin{array}{c}0.000383 \\
(20.584821)\end{array}$ \\
\hline Energía & $\begin{array}{c}14 \\
(26.4 \%)\end{array}$ & $\begin{array}{c}24 \\
(45.3 \%)\end{array}$ & $\begin{array}{c}15 \\
(28.3 \%)\end{array}$ & $\begin{array}{c}13 \\
(24.5 \%)\end{array}$ & $\begin{array}{c}40 \\
(75.5 \%)\end{array}$ & $\begin{array}{l}0 \\
(0 \%)\end{array}$ & $\begin{array}{c}0.173072 \\
(3.508095)\end{array}$ \\
\hline
\end{tabular}


estrechamente en la semana 32.

El número de mujeres que presentaron anemia al inicio del estudio disminuyó la frecuencia al finalizar el estudio. No se encontró una significancia estadística para ningún indicador bioquímico.

\section{DISCUSIÓN}

El sobrepeso y obesidad gestacional son un problema de salud mundial que implican dislipidemia, diabetes gestacional, hipertensión arterial, macrosomia, falla en la lactancia, complicaciones durante el trabajo de parto y retención de peso postparto, lo que genera riesgo tanto para la mujer embarazada como para el feto (5). Una revisión de González-Moreno el año 2013 en México encontró una prevalencia de obesidad en mujeres embarazadas de $11-22 \%$ (4), similar a la prevalencia de $22.5 \%$ de nuestro estudio; en datos de ENSANUT 2012 se reportó que la prevalencia de sobrepeso en mujeres de 20-49 años era $35.3 \%$ y $35.2 \%$ de obesidad, cifras elevadas para lo encontrado en el presente estudio. El promedio de IMC pregestacional fue $28.5 \mathrm{~kg} / \mathrm{m} 2$, a diferencia de lo mostrado por Tomedi en su estudio con mujeres embarazadas americanas donde el IMC pregestacional fue $26.6 \mathrm{~kg} / \mathrm{m} 2$ (18), lo cual se relaciona con los incrementos alarmantes de casos de sobrepeso y obesidad en nuestro país (2).

Con la implementación de la dieta personalizada durante tres meses acompañada de una orientación alimentaria previa, hubo un impacto positivo en los patrones de alimentación, en este estudio se encontró un incremento de $30.2 \%$ en la adecuación de energía recomendada de 90-110\%, similar a lo reportado por Lee y colaboradores en Australia donde el porcentaje de mejora fue $28 \%$ (19). Al finalizar el estudio ninguna paciente sobrepasó los niveles de adecuación de energía, contrario a los reportes de Cohen y colaboradores en Canadá donde $54 \%$ de las mujeres con un plan de alimentación individualizado excedieron su ingesta diaria de calorías correspondiente (20).

Los alimentos que mejoraron su consumo son las frutas, verduras y azúcares, y aunque no hubo diferencia significativa en varios alimentos, se deduce que las pacientes mejoraron principalmente sus porciones de comida, grasas y azúcares, reflejado en porcentajes de adecuación de energía y macronutrimentos correctos. Un estudio realizado en Holanda de frecuencia de consumo de alimentos sin intervención dietética, reportó que $81 \%$ de las mujeres embarazadas consumían frutas diariamente y $85.3 \%$ verduras (21), a diferencia de lo encontrado en este estudio donde al inicio $77.4 \%$ y $33.9 \%$ consumían diariamente frutas y verduras respectivamente, siendo este último donde se observó mayor diferencia, lo que se relaciona con el factor cultural en nuestro país donde hay una preferencia por alimentos con alto valor calórico, azúcares y grasas, y poco cuidado por la salud.

Una de las limitaciones de este estudio fue en la medición de la actividad física, aunque se sugirió la realización de ésta; y se mostraron cambios significativos en el sedentarismo de las

\section{TABLA 3}

Porcentaje de grupos de alimentos que consumen las pacientes al inicio y al final del estudio.

\begin{tabular}{|c|c|c|c|c|c|}
\hline \multirow[t]{2}{*}{ Grupos de alimentos } & \multicolumn{2}{|c|}{ Inicio } & \multicolumn{2}{|c|}{ Final } & \multirow{2}{*}{$\begin{array}{c}\mathrm{p} \\
\left(x^{2} \text { McNemar }\right)\end{array}$} \\
\hline & $\mathrm{n}$ & $\%$ & $\mathrm{n}$ & $\%$ & \\
\hline Frutas & 41 & 77.4 & 49 & 92.4 & 0.0047 \\
\hline Verduras & 18 & 33.9 & 32 & 60.4 & 0.0002 \\
\hline Lácteos & 29 & 54.7 & 34 & 64.1 & 0.0253 \\
\hline Leguminosas & 31 & 58.5 & 22 & 41.5 & 0.0027 \\
\hline Cereales con grasa & 12 & 22.6 & 10 & 18.9 & 0.1573 \\
\hline Cereales sin grasa & 53 & 100 & 52 & 98.1 & 0.3000 \\
\hline AOA con muy bajo o bajo aporte de grasa & 24 & 45.3 & 28 & 52.8 & 0.0455 \\
\hline AOA con moderado aporte de grasa & 21 & 39.6 & 23 & 43.4 & 0.1573 \\
\hline AOA con alto aporte de grasa & 11 & 20.7 & 9 & 16.9 & 0.1573 \\
\hline Aceites y grasas & 44 & 83 & 48 & 90.6 & 0.0455 \\
\hline Azúcares & 47 & 88.7 & 39 & 73.6 & 0.0047 \\
\hline
\end{tabular}

TABLA 4

Actividad física realizada por los pacientes al inicio y al final del estudio.

\begin{tabular}{lcccccc}
\hline Actividad física & & Inicio & & & Final & $X^{2}$ \\
& $\mathrm{n}$ & $\%$ & $\mathrm{n}$ & $\%$ & 18.9 & \\
Sedentaria & 23 & 43.4 & 10 & 26.4 & 0.012410 \\
Poco activa & 18 & 33.9 & 29 & 54.7 & \\
Activa & 12 & 22.6 & 29 & \\
\hline
\end{tabular}


pacientes, la medición fue subjetiva de acuerdo a lo que ellas reportaban, donde caminar fue la actividad física predominante al igual que en un estudio en Australia sobre comportamientos de las mujeres embarazadas (22). Con los resultados obtenidos se muestra una diferencia con lo reportado en un estudio observacional en China, donde se mostró que la población se centraba en la clasificación de activa con $43.4 \%$ (23) contra $54.7 \%$ en el presente estudio; este mismo estudio en mujeres chinas mostraba que las mujeres activas tenían una ganancia de peso menor que las mujeres sedentarias (23).

El consumo de alimentos ricos en calorías pero nutritivamente pobres y la inactividad física son preocupantes para la salud de todas las personas, pero afecta de manera desproporcionada a las madres gestantes y sus hijos, afortunadamente el peso es un factor de riesgo modificable (24). El modelo más utilizado para promover una ganancia de peso adecuado de acuerdo al IMC pregestacional es el propuesto por el Instituto de Medicina de Estados Unidos (IOM), sin embargo, en el estudio en el que está basado sólo se estudiaron a mujeres con peso adecuado pregestacional cuyos resultados sólo pueden ser extrapolados a mujeres con un estado nutricional satisfactorio, motivo por el cual se tomó como referencia las tablas de Casanueva y col. establecidas para la población mexicana y en el que se estudiaron a embarazadas sanas que alcanzaron buenos resultados en su embarazo,(25). Debido a que no hubo una adherencia del 100\% al plan de alimentación otorgado, no se logró alcanzar el peso ideal en las pacientes de acuerdo a las semanas de gestación, sin embargo, se logró controlar este peso, reflejado en que no sobrepasó el peso real al peso proyectado. Un estudio en Canadá en mujeres con sobrepeso u obesidad con un plan de alimentación individualizado reportó un promedio de aumento de peso de $0.7 \mathrm{~kg}$ por semana (20), en comparación con $1.8 \mathrm{~kg}$ en promedio de ganancia de peso en nuestras pacientes en un mes.

Ninguna de las participantes en este estudio fue detectada con DMG. Al finalizar el estudio hubo una mejora de $9.1 \%$ en los niveles de anemia, lo que puede estar relacionado con que las pacientes iniciaron en el segundo trimestre donde hay anemia fisiológica y varias terminaron en el tercer trimestre donde hay una recuperación. Por otro lado, el $24.9 \%$ de las pacientes padecía anemia al finalizar el estudio, porcentaje menor en comparación a un estudio realizado en Brasil donde $53.6 \%$ de las pacientes la padecía (26), lo que puede estar relacionado con el hierro o sulfato ferroso que se les indicó a todas las pacientes.

Debe prestarse especial atención a la ganancia de peso pregestacional, Shub y colaboradores refieren que muchas mujeres subestiman la importancia de este peso, saben que tienen obesidad pero no su IMC y las complicaciones que conlleva (27). Los profesionales de la salud tienen un papel importante en el asesoramiento y apoyo a las mujeres embarazadas (22), si bien la atención está centrada en la obstetricia, debe complementarse con estilos de vida saludable que englobe dieta sana, actividad física regular y automonitoreo del peso corporal (28); Walsh y colaboradores refieren que mujeres que recibieron intervención dietética tuvieron una ganancia de peso menor de manera significativa $(p=0.01)$ cuando se compararon con mujeres que no la tuvieron, 12.2 y $13.7 \mathrm{~kg}$ respectivamente a

\section{TABLA 5}

Medianas de las variables antropométricas de las pacientes de acuerdo a las semanas de gestación y de acuerdo a peso ideal, proyectado y real.

\begin{tabular}{|c|c|c|c|c|c|c|c|c|}
\hline \multirow{2}{*}{$\begin{array}{l}\text { No. de } \\
\text { evaluación }\end{array}$} & \multirow{2}{*}{$\begin{array}{c}\text { Peso / } \\
\text { Semanas } \\
\text { de gestación }\end{array}$} & \multicolumn{2}{|c|}{ Ideal } & \multicolumn{2}{|c|}{ Real } & \multicolumn{2}{|c|}{ Proyectado } & \multirow{2}{*}{$\stackrel{p}{\mathrm{p}}$} \\
\hline & & mdn & RIQ & Mdn & RIQ & Mdn & RIQ & \\
\hline \multirow[t]{5}{*}{ Primera } & $16(n=37)$ & 73.4 & 55.0 & 72.4 & 56.41 & 73.9 & 56.57 & 0.974733 \\
\hline & $18(n=1)$ & 70.3 & 3 & 68.6 & 2 & 66.9 & 1 & 0.367879 \\
\hline & $20(n=11)$ & 74.7 & 15.8 & 73.5 & 17.64 & 78.8 & 17.55 & 0.883839 \\
\hline & $24(n=1)$ & 81.4 & 1 & 84.1 & 2 & 86.9 & 3 & 0.367879 \\
\hline & $26(n=3)$ & 84.8 & 3.67 & 96.1 & 4.67 & 98.6 & 6.67 & 0.393241 \\
\hline \multirow[t]{5}{*}{ Segunda } & $20(n=37)$ & 74.2 & 54.1 & 75.6 & 55.78 & 75.9 & 58.16 & 0.859043 \\
\hline & $22(n=1)$ & 71.2 & 1 & 72 & 2 & 72.8 & 3 & 0.367879 \\
\hline & $24(n=11)$ & 74.4 & 16.1 & 73.8 & 17.55 & 77.5 & 17.36 & 0.928770 \\
\hline & $28(n=1)$ & 82.1 & 1 & 85.3 & 2 & 88.6 & 3 & 0.367879 \\
\hline & $0(n=3)$ & 85.5 & 3.3 & 99.1 & 5 & 100.7 & 6.67 & 0.329193 \\
\hline \multirow[t]{5}{*}{ Tercera } & $24(n=37)$ & 74.9 & 52.3 & 76.7 & 56.11 & 77.0 & 59.57 & 0.625786 \\
\hline & $26(n=1)$ & 72.2 & 1 & 76.6 & 2 & 81.3 & 3 & 0.367879 \\
\hline & $28(n=11)$ & 75.1 & 15 & 74 & 17.18 & 77.5 & 18.82 & 0.649406 \\
\hline & $32(n=1)$ & 82.9 & 1 & 86.8 & 2 & 90.9 & 3 & 0.367879 \\
\hline & $34(n=3)$ & 86.2 & 3.3 & 99.8 & 5 & 102.0 & 6.67 & 0.329193 \\
\hline \multirow[t]{5}{*}{ Cuarta } & $28(n=37)$ & 75.6 & 50.2 & 77.6 & 55.92 & 78.9 & 61.84 & 0.301048 \\
\hline & $30(n=1)$ & 73.1 & 1 & 80.6 & 2 & 88.9 & 3 & 0.367879 \\
\hline & $32(n=11)$ & 75.9 & 14.7 & 75.6 & 17.36 & 79.9 & 19 & 0.564561 \\
\hline & $36(n=1)$ & 83.6 & 1 & 87.8 & 2 & 92.2 & 3 & 0.367879 \\
\hline & $38(n=3)$ & 87.0 & 3.3 & 100.3 & 5 & 106.0 & 6.67 & 0.329193 \\
\hline
\end{tabular}




\section{GRÁFICA 1}

Promedios de los pesos de las pacientes de acuerdo a las semanas de gestación n=37.

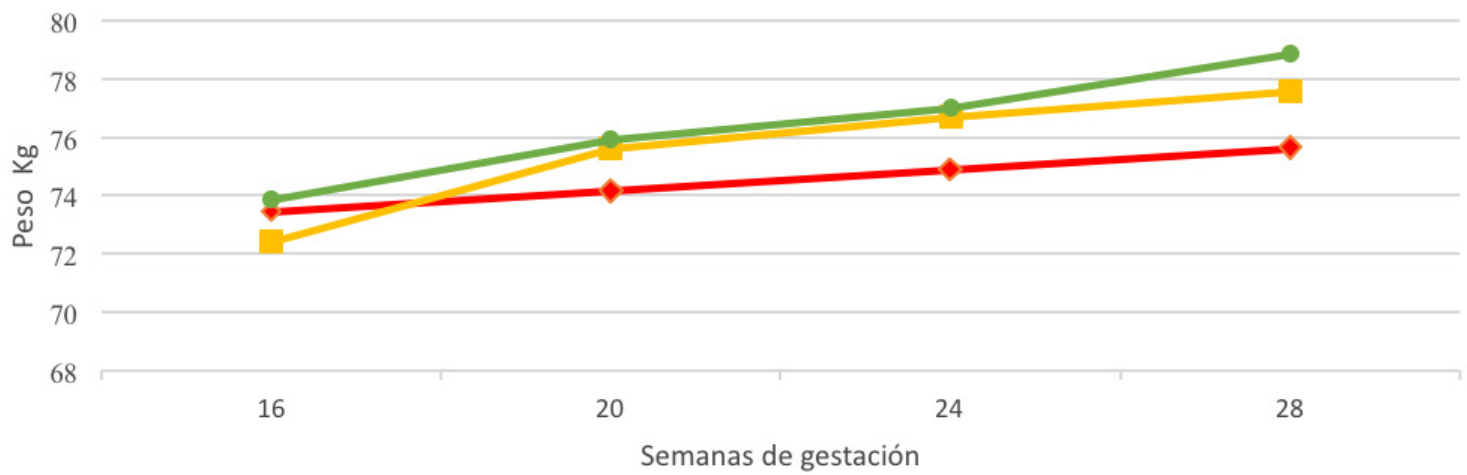

- - ideal $=$ real $\longrightarrow$ proyectado

\section{GRÁFICA 2}

Promedios de los pesos de las pacientes de acuerdo a las semanas de gestación $n=11$.

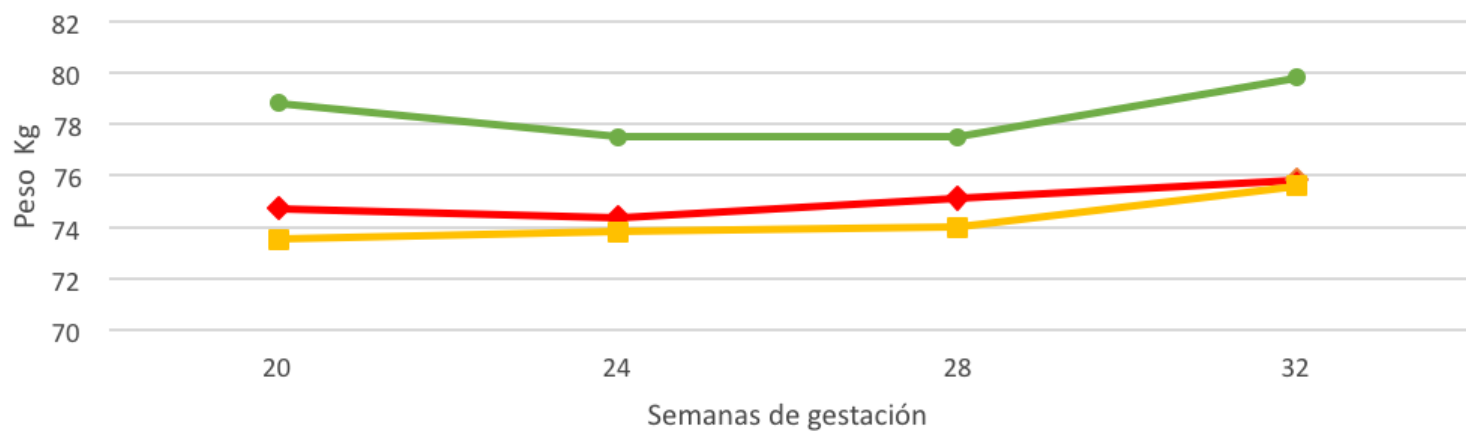

$\longrightarrow$ - - ideal $\longrightarrow$ real $\longrightarrow$ proyectado

\section{TABLA 6}

Porcentaje de pacientes con variables bioquímicas controladas al inicio y al final del estudio.

\begin{tabular}{|c|c|c|c|c|c|}
\hline \multirow[t]{2}{*}{ Bioquímicos } & \multicolumn{2}{|c|}{ Inicio } & \multicolumn{2}{|c|}{ Final } & \multirow[t]{2}{*}{$\mathrm{p}\left(\mathrm{x}^{2} \mathrm{McNemar}\right)$} \\
\hline & $\mathrm{n}$ & $\%$ & $\mathrm{n}$ & $\%$ & \\
\hline Hemoglobina normal & 35 & 66 & 40 & 75.5 & 0.3173 \\
\hline Hematocrito normal & 45 & 84.9 & 51 & 96.2 & 0.0578 \\
\hline Glucosa normal & 46 & 86.8 & 41 & 77.4 & 0.2253 \\
\hline
\end{tabular}


las 40 semanas de gestación (8). Lee y colaboradores exponen algunas sugerencias que dieron mujeres embarazadas para una mejor intervención que incluyen: demostraciones de cocina y recetas, consejos para el control de peso postparto, mayor intensidad en el ejercicio, guía de nutrición para la lactancia, higiene de alimentos y un programa de alimentación (19).

\section{CONCLUSIÓN}

La implementación de una dieta personalizada mejora el estado nutricio de las mujeres embarazadas con sobrepeso u obesidad. El principal indicador que mejoró fue el dietético, donde hubo cambios en los patrones de alimentación, y la ganancia de peso gestacional se controló. El aumento de peso excesivo durante el embarazo se asocia con un mayor riesgo de complicaciones para la madre y el hijo, los resultados de este estudio demuestran que el estado nutricio de la mujer embarazada puede mejorar con una alimentación adecuada, actividad física, apoyo y seguimiento de un nutriólogo.

\section{RESUMEN}

Objetivo: Determinar si la implementación de una dieta personalizada durante tres meses, mejora el estado nutricional de mujeres embarazadas con sobrepeso y obesidad de la UMF No. 56. Metodología: Estudio prospectivo longitudinal en 53 embarazadas con sobrepeso u obesidad a las que se les proporcionó una dieta específica, de acuerdo a sus características particulares; se evaluaron las características de la dieta correcta, la $\mathrm{R} 24 \mathrm{H}$, la frecuencia de consumo de alimentos, el peso corporal e indicadores bioquímicos. Se comparó el peso real, peso proyectado y peso ideal de las pacientes. Se aplicó prueba de McNemar y Kuskal-Wallis con nivel de confianza de 95\%. Resultados: el promedio del IMC pregestacional fue $28.5 \mathrm{~kg} / \mathrm{m} 2$. Al finalizar el estudio las gestantes mejoraron las características de la dieta correcta: la completa $(9.4 \%$ a $60.4 \%)$, el porcentaje recomendable de adecuación de lípidos (7.5\% a 45.3\%) y aumentaron la actividad física activa (22.6\% a 54.7\%). No se alcanzó el peso ideal gestacional, pero se logró control de peso, reflejado en peso proyectado mayor al real en cada intervención. Sin cambios significativos en el indicador bioquímico ( $p>0.05)$. Conclusiones: La implementación de la dieta personalizada mejoró el estado nutricional de mujeres embarazadas con sobrepeso u obesidad en sus patrones de alimentación y en el control de peso gestacional.

Palabras clave: ganancia de peso gestacional; sobrepeso; obesidad; embarazo; peso corporal.

\section{BIBLIOGRAFÍA}

1. WHO. Obesity and overweight [Internet]. 2015 [Updated January 2015; Accessed February 18th, 2015]. Available in: http://www.who.int/mediacentre/factsheets/fs311/es/

2. Instituto Nacional de Salud Pública. Encuesta Nacional de Salud y Nutrición [Internet]. 2012 [Updated 2012; Accessed February 18th, 2015]. Available in: http:// ensanut.insp.mx/informes/ENSANUT2012ResultadosNacionales.pdf

3. Brown, J. Nutrición durante el embarazo. In: Brown J. Nutrición en las diferentes etapas de la vida. 3ed. Mexico: McGraw Hill; 2008. p. 83-128.

4. Institute of Medicine. Weight gain during pregnancy: reexamining the guidelines [Internet]. 2009 [Updated May 2009; Accessed February 18th, 2015]. Available in: http:// iom.edu/ /media/Files/Report\%20Files/2009/WeightGain-During-Pregnancy-Reexamining-the-Guidelines/
Report\%20Brief\%20-\%20Weight\%20Gain\%20During\%20Pregnancy.pdf

5. González-Moreno J, Salvador J, Rodríguez J. Pregnancy Obesity. MD. 2013; 4: 270-5.

6. Zonana-Nacach A, Baldenebro-Preciado R, Ruiz-Dorado $M$. The effect of gestational weight gain on maternal and neonatal outcomes. Salud Publica Mex. 2010. 52 (3); 220-5.

7. Silva-del Valle M, Sánchez-Villegas A, Serra-Majem L. Association between the adherence to the Mediterranean diet and overweight and obesity in pregnant women in Gran Canaria. Nutr Hosp. 2013; 28(3): 654-9.

8. Walsh J, McAuliffe. Impact of maternal nutrition on pregnancy outcome - Does it matter what pregnant women eat?. Best Pract Res Clin Obstet Gynaecol. 2014; 29: 63-78.

9. Renault K, Carlsen E, Norgaard K, Nilas L, Pryds O, Secher $N$, et al. Intake of sweets, snacks and soft drinks predict weight gain in obese pregnant women: detailed analysis of the results of a randomised controlled trail. PLoS One. 2015; 10: 1-15.

10. Agha M, Agha R, Sandell J. Conceptual and pregnant women. A systematic review and meta-analysis. PLos ONE. 2014; 9 (5): 1-16.

11. Sydsj G, Monfils W, de Keyser N, Claesson I, Sydsj A, Josefsson $A$. Effects of a weight-gain restriction programme for obese pregnant women on sickness absence and pregnancy benefits. Scand J Prim Health Care. 2013; 31(2): 106-10.

12. Secretaria de Salud. Norma Oficial Mexicana NOM-043SSA2-2012, servicios básicos de salud. Promoción y educación para la salud en materia alimentaria. Criterios para brindar orientacion. [Internet]. Mexico D.F.: Secretaria de gobernación; 2012. [Updated January 22, 2013; Accessed March 21, 2015] Available in: http://www.dof.gob.mx/ nota_detalle.php?codigo $=5285372 \&$ fecha $=22 / 01 / 2013$

13. Lohman T. Antropometric Standarization Reference Manual.

14. Palafox $M$, Ledesma J. Pregnant woman. In: Palafox $M$, Ledesma J. Nutritional intervention formula manual. 2 ed. Mexico: McGraw-Hill; 2012. p. 1-14.

15. Casanueva E, Flores-Quijano M. Nutrition of the adult woman. In: Casanueva E, Kaufer M, Perez A. Medical Nutriology. 3a ed. Mexico: Panamericana; 2008. p. 697.

16. Haua K. D: Nutrition: evaluation strategies. In: Suverza $A$, Haua $K$. The $A B C D$ assessment of nutrition status. 1ed. México: McGraw-Hill; 2010. p. 225-252

17. Ayoubi J, Hirt R, Badiou W, Hininger-Favier I, Favier M, Zraik-Ayoubi $F$, Berrebi $A$, et al. Nutrition in pregnant women. EMC. 2012; 48 (2): 1-14.

18. Tomedi L, Luther J, Ho Chung-Chou, Evans R, Simhan $H$, Wisner $K$, et al. The association between pre-pregnancy obesity and maternal nutritional biomarker status during pregnancy: a factor analysis. Public Health Nutr. 2013; 16 (8): 1414-8.

19. Lee A, Karpavicius J, Gasparini E, Forster D. Implementing a diet and exercise program for limiting maternal weight gain in obese pregnant women: a pilot study. Aust N Z J Obstet Gynaecol. 2012; 52(5): 427-32.

20. Cohen T, Koski K. Limiting excess weight gain in healthy pregnant women: importance of energy intakes physical activity, and adherence to gestational weight gain guidelines. J Prenancy. 2013; 1-13.

21. Baron R, Manniën J, Velde S, Klomp T, Hutton E, Brug J. Socio-demographic inequalities across a range of health status indicators and health behaviours among pregnant women in prenatal primary care: a cross-sectional study. 
BMC Pregnancy Childbirth. 2015; 15 (261): 1-11.

22. Jersey S, Nicholson J, Callaway L, Daniel L. An observational study of nutrition and physical activity behaviours, knowledge, and advice in pregnancy. BMC Pregnancy Childbirth. 2013; 13 (115): 1-8.

23. Jiang $H$, Qian X, Li M, Lynn H, Fan YJiang $H$, et al. Can physical activity reduce excessive gestational weight gain? Findings from a Chinese urban pregnant women cohort study. Int J Behav Nutr Phys Act. 2012; 9 (12): 1-7.

24. Dean S, Lassi Z, Imam A, Bhutta Z. Preconception care: nutritional risks and interventions. Reprod Health. 2014; 11 (3): 1-15.

25. Casanueva E, Flores Quijano M.E., Roselló-Soberón M.E., De-Regil L.M., Sámano R. Gestational weight gain as predicted by pregestacional body mass index and gesta- tional age in Mexican women. Food Nutr Bull. 2008; 29 (4): 334-9.

26. Augusta S, Willner E, Aguiar T, Rosse V, Teles G, Blondet $V$. Anemia in pregnancy: impact on weight and in the development of anemia in newborn. Nutr Hosp. 2015; 32 (5): 2071-9.

27. Shub A, Huning E, Campbell K, McCarthy E. Pregnant women's knowledge of weight, weight gain, complications of obesity and weight management strategies in pregnancy. BMC Res Notes. 2013; 6(1): 1-6.

28. Rauh K, Kunath J, Rosenfeld E, Kick L, Ulm K, Hauner H. Healthy living in pregnancy: a cluster-randomized controlled trial to prevent excessive gestational weight gain - rationale and design of the GeliS study. BMC Pregnancy Childbirth. 2014; 14 (119): 1-9.

\begin{tabular}{|c|c|}
\hline SEGURIDAO Y SOLIDARIOAO SOCLAL & $\begin{array}{l}\text { NSTITUTO MEXICANO DEL SEGURO SOCIAL } \\
\text { UNIDAD DE EDUCACIÓN, INVESTIGACIÓN } \\
\text { Y POLITICAS DE SALUD } \\
\text { OORDINACIÓN DE INVESTIGACIÓN EN SALUD } \\
\text { CARTA DE CONSENTIMIENTO INFORMADO } \\
\text { (ADULTOS) }\end{array}$ \\
\hline \multicolumn{2}{|c|}{ CARTA DE CONSENTIMIENTO INFORMADO PARA PARTICIPACIÓN EN PROTOCOLOS DE INVESTIGACIÓN } \\
\hline Nombre del estudio: & $\begin{array}{l}\text { Efecto de una dieta personalizada en la mejora del estado nutricio en mujeres } \\
\text { embarazadas con sobrepeso y obesidad }\end{array}$ \\
\hline \multicolumn{2}{|l|}{ Patrocinador externo (si aplica): } \\
\hline Lugar y fecha: & UMF NO. 56 León, Guanajuato. A \\
\hline \multicolumn{2}{|l|}{ Número de registro: } \\
\hline Justificación y objetivo del estudio: & $\begin{array}{l}\text { El sobrepeso y obesidad son una de las principales causas de complicaciones durante el } \\
\text { embarazo tanto para la madre como para el feto, lo cual representa un grave problema de } \\
\text { salud pública. Con la realización de este estudio de espera mejorar el estado nutricio de } \\
\text { la mujer embarazada, a través de una adecuada ganancia de peso gestacional, así como } \\
\text { que se reduzcan el riesgo de complicaciones durante y al final del embarazo. }\end{array}$ \\
\hline Procedimientos: & $\begin{array}{l}\text { Llenado de la historia clínico-nutricia del paciente en la primera consulta. Se aplicará un } \\
\text { recordatorio de } 24 \text { horas, frecuencia de consumo de alimentos y medición de peso y talla } \\
\text { para evaluar cambios. Al inicio y al final del estudio se realizará la paciente un análisis } \\
\text { sanguíneo de glucosa, hemoglobina y hematocrito. Al finalizar cada consulta se le dará a } \\
\text { la paciente un plan de alimentación de acuerdo a sus necesidades energéticas. }\end{array}$ \\
\hline Posibles riesgos y molestias: & $\begin{array}{l}\text { Podría llegar a haber molestias durante la medición del peso y la talla ya que deben de } \\
\text { ser con la menor de ropa posible. La toma de análisis sanguíneo es un método invasivo. }\end{array}$ \\
\hline $\begin{array}{l}\text { Posibles beneficios que recibirá al participar } \\
\text { en el estudio: }\end{array}$ & $\begin{array}{l}\text { Mejora del estado nutricio reflejado en una adecuada ganancia de peso gestacional, } \\
\text { hábitos de alimentación e índices bioquímicos. Reducción del riesgo de complicaciones } \\
\text { durante y al final del embarazo. }\end{array}$ \\
\hline
\end{tabular}




\section{CONTINUACIÓN ANEXO 1}

Información sobre resultados y alternativas de tratamiento:

Participación o retiro:

Privacidad y confidencialidad:
En el transcurso del estudio usted podrá solicitar información actualizada sobre el mismo al investigador responsable.

Si decide participar en el estudio puede retirarse en el momento que lo desee, aun cuando el investigador responsable no se lo solicite, pudiendo informar o no, las razones de su decisión, la cual será respetada en su integridad. El abandonar el estudio no afectara su derecho a recibir atención médica en el Instituto.

La información obtenida en este estudio, utilizada para la identificación de cada paciente, será mantenida con estricta confidencialidad y solo el investigador responsable tendrá acceso a esta información.

En caso de colección de material biológico (si aplica):

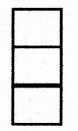

No autoriza que se tome la muestra.

Si autorizo que se tome la muestra solo para este estudio.

$\mathrm{Si}$ autorizo que se tome la muestra para este estudio y estudios futuros.

Disponibilidad de tratamiento médico en derechohabientes (si aplica):

Beneficios al término del estudio:

Adecuado estado nutricio con reduccion de complicaciones durante y al finalizar el embarazo.

En caso de dudas o aclaraciones relacionadas con el estudio podrá dirigirse a:

Investigador Responsable:

PSSN Karen Vianey Sandoval García Tel. 4621095646 Correo: bash_kv@hotmail.com

Colaboradores:

Dr. Efrén Nieves Correo: efren.nieves@imss.gob.mx Tel. 4777781110

Dr. Miguel Ángel Luna Ruíz Correo: lunaruizmiguelangel91@gmail.com

En caso de dudas o aclaraciones sobre sus derechos como participante podrá dirigirse a: Comisión de Ética de Investigación de la CNIC del IMSS: Avenida Cuauhtémoc $3304^{\circ}$ piso Bloque "B" de la Unidad de Congresos, Colonia Doctores. México, D.F., CP 06720. Teléfono (55) 56276900 extensión 21230, Correo electrónico: comision.etica@imss.gob.mx

Nombre y firma del sujeto

Testigo 1
Nombre y firma de quien obtiene el consentimiento

Testigo 2

Nombre, dirección, relación y firma

Nombre, dirección, relación y firma

Este formato constituye una guía que deberá completarse de acuerdo con las características propias de cada protocolo de investigación, sin omitir información relevante del estudio

Clave: 2810-009-013 


\section{Datos personales}

Paciente No:

NSS:

Fecha:

Nombre:

Fecha de nacimiento:

Estado civil:

Edad:

Ocupación:

Domicilio:

Teléfono: ( )

Escolaridad:

\begin{tabular}{|c|c|c|c|}
\hline \multicolumn{2}{|c|}{$\begin{array}{l}\text { Antecedentes } \\
\text { Heredo-familiares }\end{array}$} & $\begin{array}{c}\text { Antecedentes } \\
\text { Personales Patológicos }\end{array}$ & \multirow{2}{*}{$\begin{array}{l}\begin{array}{l}\text { Antecedentes Gineco- } \\
\quad \text { Obstétricos }\end{array} \\
\text { Edad de menarca: } \\
\text { No. Embarazos: } \\
\text { No. partos: } \\
\text { No. cesáreas: } \\
\text { Abortos: }\end{array}$} \\
\hline $\begin{array}{l}\text { ( ) Obesidad } \\
\text { ( ) HTA } \\
\text { ( ) Dislipidemias } \\
\text { ( ) Pad. Renal } \\
\text { ( ) Osteoporosis } \\
\text { ( ) Pad. Cardio } \\
\text { ( ) Otros }\end{array}$ & $\begin{array}{l}\text { ( ) Diabetes } \\
\text { ( ) Infarto } \\
\text { ( ) Litiasis } \\
\text { ( ) Pad. } \\
\text { Digestivos } \\
\text { ( ) Cáncer }\end{array}$ & $\begin{array}{l}\text { ( ) Obesidad } \\
\text { ( ) Sobrepeso } \\
\text { ( ) Diarrea } \\
\text { ( ) Dislipidemia } \\
\text { ( ) Estreñimiento } \\
\text { ( ) Gastritis } \\
\text { ( ) Cirugías } \\
\text { ( ) Alergias a alimentos }\end{array}$ & \\
\hline \multicolumn{4}{|c|}{ Antecedentes Personales No Patológicos } \\
\hline $\begin{array}{l}\text { Tipo: } \\
\text { Cantidad: }\end{array}$ & entos que ingiere: & $\begin{array}{l}\text { Actividad Física: } \\
\text { Tipo: } \\
\text { Duración: }\end{array}$ & \\
\hline
\end{tabular}

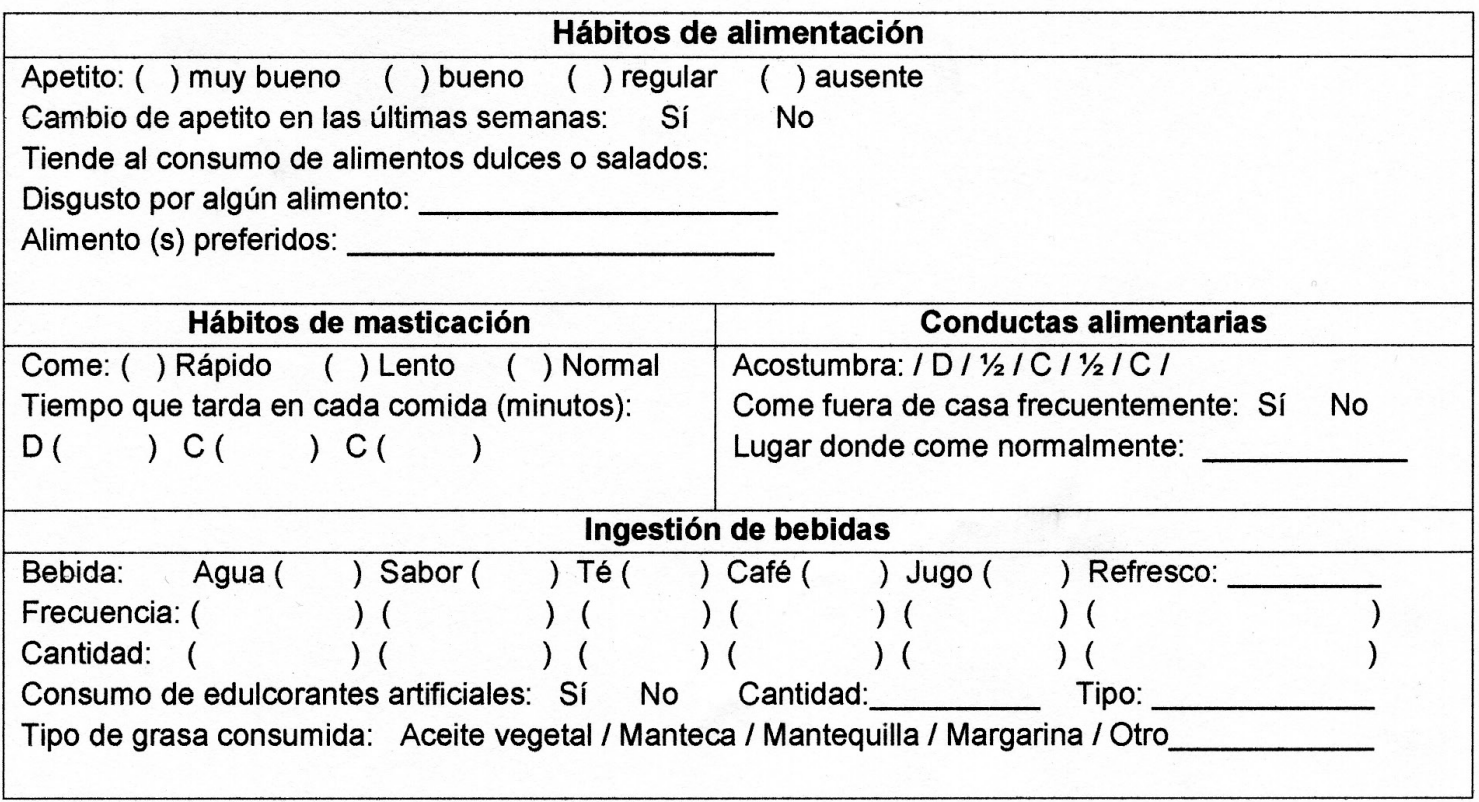


ANEXO 3

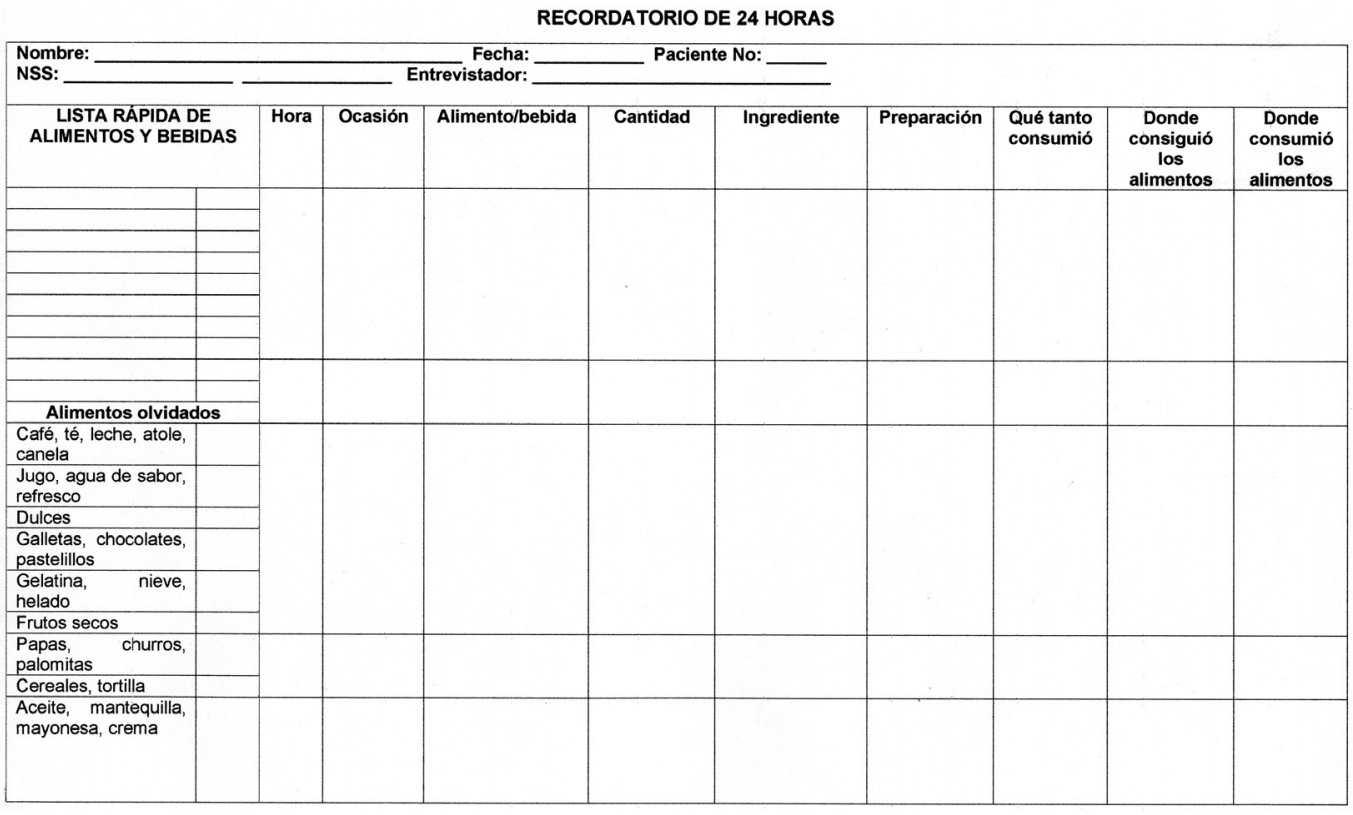

FRECUENCIA DE ALIMENTOS

\begin{tabular}{|l|l|l|l|l|l|}
\hline \multicolumn{1}{|c|}{ ALIMENTO } & FRECUENCIA & \multicolumn{1}{|c|}{ ALIMENTO } & FRECUENCIA & \multicolumn{1}{c|}{ ALIMENTO } & FRECUENCIA \\
\hline Tortilla & Huevo & & Carne de res & \\
\hline Arroz & & Leguminosas & & Carne de puerco & \\
\hline Tamales & Aceite & & Pollo & \\
\hline Bolillo & Manteca & & Pescado & Salchicha \\
\hline Pan dulce & Mantequilla & & Jamón & \\
\hline Pan blanco & Aguacate & & Menudo & \\
\hline Avena & Margarina & & Chorizo & \\
\hline Papa & Azúcar & & Leche & \\
\hline Pastas & Refresco & & Crema & \\
\hline Galletas & Jugo & & Queso & \\
\hline Yogurt & Condimentos & & Otros & \\
\hline Frutas & Verduras & & & \\
& & & & & \\
\hline
\end{tabular}

ANEXO 4

ANTROPOMETRÍA

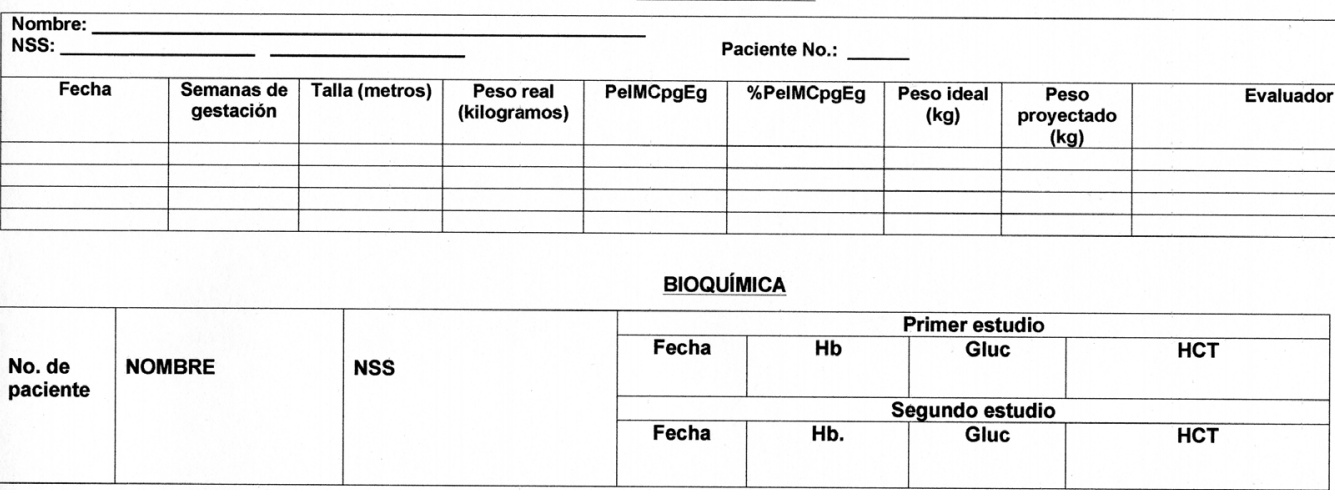




\section{ANEXO 5}

Aumento de peso durante el embarazo. Población mexicana. Casanueva colaboradores, 2008.

\begin{tabular}{|c|c|c|c|c|}
\hline \multirow[b]{2}{*}{$\begin{array}{l}\text { Edad de } \\
\text { gestación } \\
\text { (semana) }\end{array}$} & \multicolumn{4}{|c|}{ Aumento de peso (k9) } \\
\hline & $\begin{array}{c}1 \mathrm{MCpg} \\
<18.5 \mathrm{~kg} / \mathrm{m}^{2}\end{array}$ & $\begin{array}{c}\text { MCDS } \\
18.5 \text { a } 24.9 \mathrm{~kg} / \\
\mathrm{m}^{2}\end{array}$ & $\begin{array}{l}\text { IMCPe } \\
25.0 \text { a } 29.9 \mathrm{~kg} \\
\mathrm{~m}^{2}\end{array}$ & $\begin{array}{l}\text { MGAI } \\
\text { 30 knim? }\end{array}$ \\
\hline 16 & 5.15 & 4.27 & 379 & 291 \\
\hline 17 & 5.47 & 4.54 & 4.03 & 311 \\
\hline 18 & 5.80 & 4.81 & 4.27 & 3.29 \\
\hline 19 & 6.12 & 5.07 & 4.50 & 348 \\
\hline 20 & 6.44 & 5.34 & 4.74 & 366 \\
\hline 21 & 6.76 & 5.61 & 4,98 & 384 \\
\hline 22 & 708 & 5.87 & 5.21 & 403 \\
\hline 23 & 7.41 & 6.14 & 5.45 & 421 \\
\hline 24 & 7.73 & 6.41 & 5.69 & 4.39 \\
\hline 25 & 8.05 & 6.68 & 5.93 & 458 \\
\hline 26 & 8.37 & 6.94 & 6.16 & 4.76 \\
\hline 27 & 8.69 & 7.21 & 6.40 & 4.94 \\
\hline 28 & 9.02 & 7.48 & 6.64 & 5.12 \\
\hline 29 & 9.34 & 7.74 & 687 & 531 \\
\hline 30 & 9.66 & 8.01 & 7.11 & 5.49 \\
\hline 31 & 9.98 & 8.28 & 7.35 & 5.67 \\
\hline 32 & 10.30 & 8.54 & 7.58 & 5.86 \\
\hline 33 & 10.63 & 8.81 & 7.82 & 6.04 \\
\hline 34 & 10.95 & 9.08 & 8.06 & 6.22 \\
\hline 35 & 11.27 & 9.35 & 8.30 & 6.41 \\
\hline 36 & 11.59 & 9.61 & 8.53 & 659 \\
\hline 37 & 1191 & 9.88 & 8.77 & 6.77 \\
\hline 38 & 1224 & 10.15 & 9.01 & 6.95 \\
\hline 39 & 12.56 & 10.41 & 9.24 & 7,14 \\
\hline 40 & 1288 & 10.68 & 9.48 & 732 \\
\hline
\end{tabular}

\section{ANEXO 6}

\section{Categoria ${ }^{21}$}

Sedentario o estilo de vida con actividad ligera: Personas con ocupación laboral que no demanda mucho esfuerzo físico. No necesitan caminar grandes distancias. Por lo general usan vehículos motorizados para transportarse. No realizan ejercicio ni participan en deportes con regularidad. La mayor parte de su tiempo libre lo pasan sentadas o paradas, con poco desplazamiento, en actividades como hablar, leer, ver la televisión, escuchar la radio o usar la computadora.

Activo o estilo de vida con actividad moderada: Personas con ocupaciones que no son extenuantes, aunque requieren más gasto de energía que la descrita para el estilo de vida sedentario. Pueden ser personas con ocupaciones sedentarias, que suelen pasar cierta parte de su tiempo en actividades físicas moderadas o vigorosas, de manera programa o circunstancial (usan más o menos una hora diaria, continua o acumulada, para trotar, correr, andar en bicicleta, bailar)

Vigoroso o estilo de vida con actividad vigoroso: Personas dedicadas de manera regular en actividades laborales o deportivas extenuantes por varias horas (actividades agrícolas no mecanizadas, natación o baile durante 2 horas diarias)

\section{Valor de NAF}

1.40 a 1.69

2.00 a 2.40 
ANEXO 7

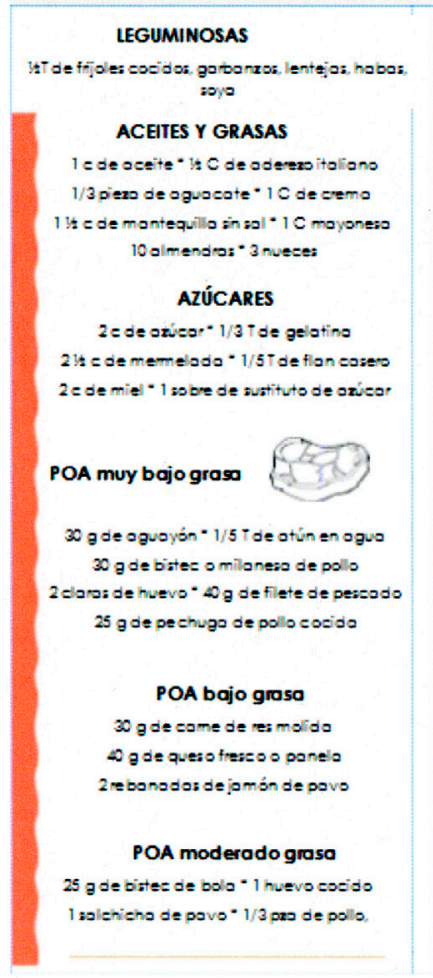

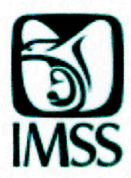

UNIDAD DE MEDICINA FAMILIAR No. 56

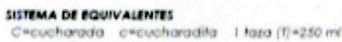

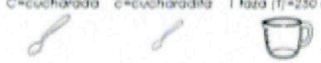

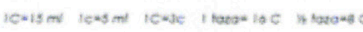

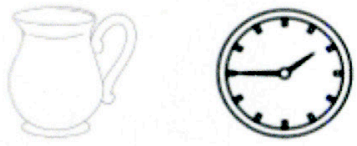

Dudes:

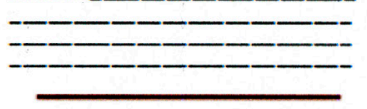

FECHA:

Pasante Nutrición. Karen Vianey Sandoval Garcia

Correoselectrónico: bash_kng/romailcom

Tot 4621095646

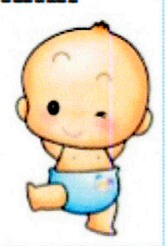

PLAN DE

AIIMENTACION

EMBARAZO

-La alegría de una madre comienza cuando una nueva vida se agita en su interior $y$ una

patadita juguetona le recuerda que ya no esta sola"

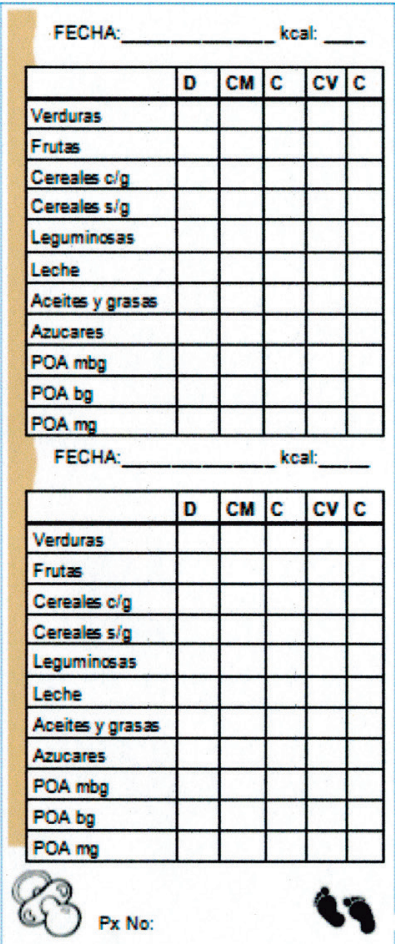

FECHA:
\begin{tabular}{|l|l|l|l|l|l|}
\hline & D & CM & C & CV & C \\
\hline Verduras & & & & & \\
\hline Frutas & & & & & \\
\hline Cereales cig & & & & & \\
\hline Cereales 5/g & & & & & \\
\hline Leguminosas & & & & & \\
\hline Leche & & & & & \\
\hline Aceites y grasas & & & & & \\
\hline Azucares & & & & & \\
\hline POA mbg & & & & & \\
\hline POA bg & & & & & \\
\hline POA mg & & & & & \\
\hline
\end{tabular}

\section{s?}

FRUTAS

1 Tde frezas rebanadaz" 1 guavaba rora 2 naranja: " 2 mandarinas" 1 mango marila 1 manxana " 1 Tde melón picado" it pero 1 Tde papayo picada " It plátono" 18 uvos 1 Tdesandía picado* 1 Tde fruta picada

10 posas $-1 / 4$ t de pina pieado - 3 cinelo:

\section{LECHE}

34 de tors de yogut light

1 tors de leche deveremado it tars de leche evoporodo

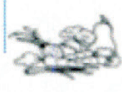

\section{VERDURAS}

14t Tde brócoli cocido" it Tde cala baxa cocida lit Tde champifiones cocidor" it T de siotes 6 espámagos ovudor" 1 jitomate It T de jicamo picodo" I T de nopales cocidos 5 tomater verde: " It T de zanohoia pieado 14 Tde col cocido picodo " 1 Tde lechugo It Tde pimiento cosido" 1 Tde verdologos

IT de acelgas* 1/2T de chayote

\section{CEREALES con graso}

34 de barita de granola quoker" 1 it tostado: it rebanada de pay de queso

1 it pro de galleta de avena" 3 C de gronsla 5 pros de galleta integral

\section{CEREALES sin graso}

14 T de amoz cocido" 34 T de avena socida 14 barita de avero * $1 / 3$ piexa de bollilo

14 Telote dergranodo $1 / 3$ T de espogueti

It Tde fides cocido" 5 galletor mań

1 rebanado de pan de caja integral 1 totillo" 36 pro de hot coke 1/4pre de wanduce 